\title{
Effect of oxygen contents on strain rate sensitivity of commercially pure titanium
}

\author{
Min-Su Lee ${ }^{1}$, Yong-Taek Hyun ${ }^{2}$, Tea-Sung Jun ${ }^{1, *}$ \\ ${ }^{1}$ Dept of Mechanical Engineering, Incheon National University, Incheon 22012, Republic of Korea \\ ${ }^{2}$ Metallic Materials Division, Korea Institute of Materials Science, Changwon 51508, Republic of Korea \\ *Corresponding author: t.jun@inu.ac.kr
}

\begin{abstract}
In this study, we have investigated the effect of oxygen contents on strain rate senstivitiy (SRS) within Gr. 1 and 4 commercially pure titanium (CP-Ti). The SRS was evaluated in multi-scales using macro-scopic tensile test with constant strain rate (CSR) method and strain rate jump (SRJ) method, and nanoindentation test with SRJ method. Electron backscatter diffraction (EBSD) has been used to characterise crystallographic texture and individual grain orientation of samples. Slip and twin activities of each CP-Ti were compared by EBSD measurements and the associated Schmid factor (SF) analysis. The active slip system is anticipated to be different in each relation between loading directions and textures, but twin activity is much similar. The texture dependent global SRS is thus thought to be resulted from the different slip activity. Local SRS was dependent not only on the grain orientation but also on the oxygen contents, leading to the fact that the impact of oxygen contents is closely correlated in macro- and micro-scopic level.
\end{abstract}

\section{Introduction}

Titanium alloys are attractive material in aerospace industry [1] due to their high strength-to-weight ratio, corrosion resistance and excellent mechanical properties. These alloys often undergo a significant cyclic loading condition during flight, and these regimes appartently have time sensitive load-hold. This cyclic loading system combined with load-hold stage is related to the fatigue life of structural components, and has shown the reduction of the number of cycles to failure by an order of magnitude or more than simple fatigue test without the holding stage [2]. This is known as dwell debit. Recent studies have devoted to unveil the dwell fatigue by exploring the rate sensitive deformation of titanium [3-8] because dwell fatigue associated with time sensitive load-hold is clearly a rate sensitive deformation mode.

Oxygen is a well-known interstitial solute that significantly affects the deformation behaviour and mechanical properties of titanium and its alloys, so that it is widely used in these materials to obtain further mechanical properties [9]. The oxygen either acts as obstacles against dislocation motion or induces lattice distorsions, thereby causing significant strengthening effect [10-12]. This solution strengthening effect by adding the oxygen contents strongly restrained the occurrence of deformation twinning [13], and the strain rate sensitivity (SRS) of titanium was significantly dependent on the volume fraction of twins [5]. However, as the significant decrease of elogation in titanium is also observed [10-12] with the oxygen cencentration, it is needed to carefully control the optimal balance between strength and ductility.

Hexagonal close-packed (HCP) metals such as titanium generally present the anisotropic deformation behaviour, and have limited numbers of slip systems compared with cubic symmetric metals [14]. There is a large difference of critical resolved shear stress (CRSS) between slip systems of titanium [15], and the CRSS value is typically increased with oxygen concentration [16]. Recent in situ micro-pillar compression test [4] showed significant slip dependent rate senstivity within planar and wavy glide on well-oriented grains for prism and basal slip activation, respectively. The transition from wavy to planar configuration of dislocation was also indentified with an increase of oxygen contents [17]. Therefore, the strain rate sensitivity of titanium is thought to be significantly different with respect to the oxygen contents.

The rate sensitive response of materials could be quantified by analysing the relation in strain rate and corresponding deformation property, such as flow stress in uniaxial tensile or compression test and hardness in indentation test, respectively. The former, as shown in Figure 1, can be obtained by conducting the constant strain rate (CRS) method and strain rate jump (SRJ) method as follows: 


$$
m=\frac{d \ln (\sigma)}{d \ln (\dot{\varepsilon})} \text { for CSR method, and } m=\frac{d \ln \left(\sigma_{2} / \sigma_{1}\right)}{d \ln \left(\dot{\varepsilon}_{2} / \dot{\varepsilon}_{1}\right)} \text { for SRJ method (1) }
$$

where, $\mathrm{m}$ is SRS exponent, $\sigma$ flow stress and $\dot{\varepsilon}$ strain rate. The different strain rate is applied on each experiment in CSR method, whilst the abrupt rate changes are created at particular strains in single experiment by SRJ method. This indicates that microstructure is remained in nearly constant state during the SRJ test. The $m$ value is determined by a plot of the flow stress and strain rate in log-log form, and then measuring the slope as shown in Figure 1(c). Note that there are transient sections in SRJ method, showing the deformation behaviour combined by prior and jumped strain rate. The data belong to this section is excluded to allow the subsistent deformation derived within each strain rate.

(a)

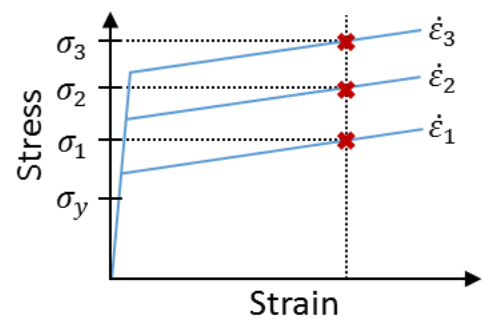

(b)

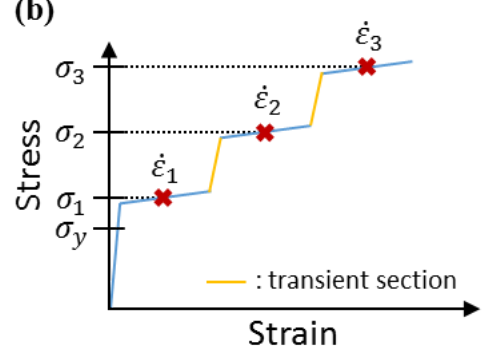

(c)

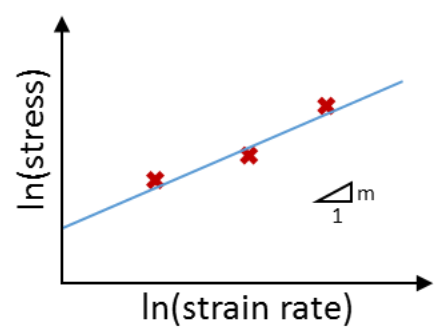

Figure 1. Schematic illustrations showing the results from the uniaxial tensile and/or compression test by (a) constant strain rate (CSR) method and (b) strain rate jump (SRJ) method, and (c) the determination of SRS exponent, $\mathrm{m}$ [18].

Nanoindentation is an effective method exploring the mechanical properties, typically in the deformation resistance (i.e. hardness) and elastic modulus on a local scale [19]. Therefore, the indentation-based SRS could be obtained in submicron level from the following equation:

$$
m=\frac{d \ln (H)}{d \ln \left(\dot{\varepsilon}_{\text {indentation }}\right)}
$$

where, the components of SRS exponent (m) are further derived as follows; hardness, $\mathrm{H}=\mathrm{P} / \mathrm{A}$ and strain rate, $\dot{\varepsilon}_{\text {indentation }}=h / h$, in which P is applied load, A projected area of indentation, h given indentation depth and $h$ the penetration ratio. In indentation test, strain rate is also determined by controlling the loading rate as follows [20]:

$$
\dot{\varepsilon}_{\text {indentation }}=\frac{\dot{h}}{h}=\frac{1}{2}\left(\frac{\dot{P}}{P}-\frac{\dot{H}}{H}\right) \approx \frac{1}{2}\left(\frac{\dot{P}}{P}\right)
$$

where, if the response of the material by indentation is steady-stated (i.e. $\dot{H}=0$ ) during the test, effective strain rate, $P / P$, can be available.

The SRJ method is applicable to the nanoindentation technique using continuous stiffness measurement (CSM) method [21]. The CSM method enables the continuous measurement of hardness as a function of depth during the indentation test by applying the sinusoidal oscillation on the force signal and measuring the response of the material. Therefore, the hardness distribution transformed by abrupt rate change could be effectively measured in indentation-based SRJ system [22] (see Figure 2(b)). 
(a)

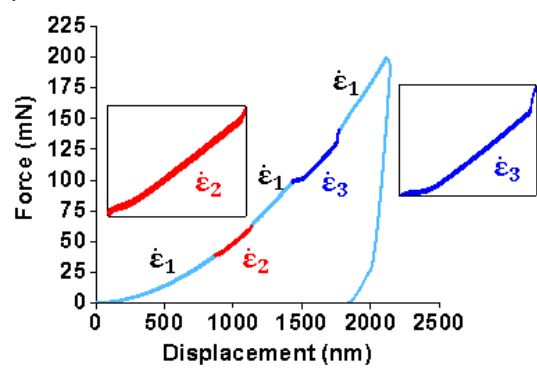

(b)

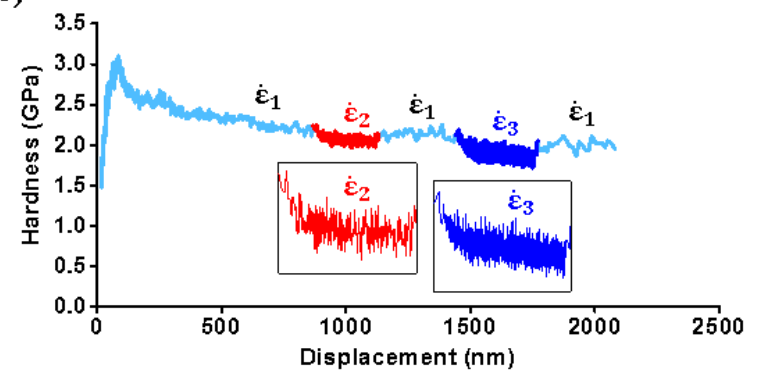

Figure 2. (a) The distribution in load vs. displacement by nanoindentation strain rate jump test with continuous stiffness measurment (CSM) method and (b) the transformed hardness as function of displacement.

Rate sensitive deformation behaviour is extensively studied in Ti alloys with micromechanical factors such as alloying component [3, 8], deformation modes [4, 6] and microstructure [7]. However, the effect of oxygen contents on strain rate sensitivity is still unclear, and the complex combination of those factors in macro-scale region confuses the understanding with respect to the rather fundamental influence. Therefore, the aim of this study is to investigate the effect of oxygen contents on SRS of titanium by conducting the macro-scopic tensile test with CSR method and SRJ method, and nanoindentation test with SRJ method in grain scale to minimize the irrelevant influence in macro-scopic level.

\section{Material and experiments}

Tensile specimens with gauge length of $25 \mathrm{~mm}$ were cut along the rolling (RD) and transverse directions (TD) from the 1-mm-thick rolled plates of Gr. 1 and $4 \mathrm{CP}-\mathrm{Ti}$, in which the oxygen contents is typically 0.18 and 0.40 wt.\% [23], respectively. The tensile tests with CSR method and SRJ method were carried out with variable strain rates (on the order of $10-2$ to $10-4 \cdot \mathrm{s}-1$ ).

Microstruture of CP-Ti was characterised by electron backscattered diffraction (EBSD) measurement. The samples were ground with $\mathrm{SiC}$ papers (up to 2400 grit) and then polished with hydrogen peroxide (i.e. $\mathrm{H} 2 \mathrm{O} 2$ ) and OP-S into a ratio of $1: 5$, respectively. It is subsequently etched with Kroll's reagent that is a mixture of $2 \% \mathrm{HF}, 10 \% \mathrm{HNO}, 88 \%$ distilled $\mathrm{H} 2 \mathrm{O}$. The polishing-etching process is repeated until the grain boundaries are clearly seen through the polarised light microscopy [24]. The EBSD measurement was carried out in a ZEISS SUPRATM 55VP FE-SEM with Bruker eSpirit v2.1 software. Probe current 120nA and accelerating voltage $20 \mathrm{kV}$ was applied. Large area maps $(1.2 \times 0.9 \mathrm{~mm} 2)$ were taken with a step size of $1.68 \mu \mathrm{m}$ for Gr. 1 and $2 \mu \mathrm{m}$ for Gr. 4, respectively.

Anton Paar nanoidenter was used to investigate the local SRS within individual grain orientations (see Figure 3) with the SRJ method. The strain rate was determined by controlling the loading rate. All of the indentation test were initiated with a strain rate of $10-1 \cdot \mathrm{s}-1$ and jumped with variable strain rates (on the order of 10-1 to 10-3.s-1) at particular displacemnet. The maximum load of $200 \mathrm{mN}$ for Gr. 1 and $300 \mathrm{mN}$ for Gr. 4 were applied so that the maximum displacement became about $2000 \mathrm{~nm}$. The CSM measurment was made with $2 \mathrm{~nm}$ amplitude and $45 \mathrm{~Hz}$ frequency. The overall procedure can be seen in Figure 2 .

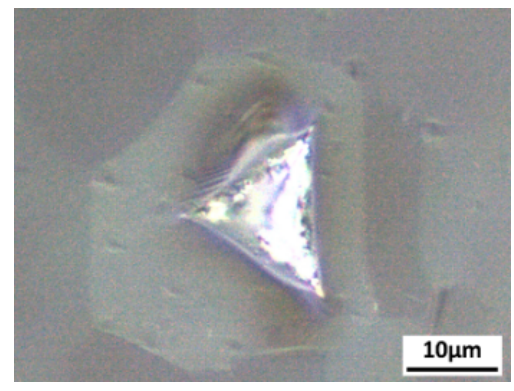

Figure 3. Representative polarised light micrope image showing the nanoindentation impression within a single grain. 
Figure 4 shows the equiaxed grain structure with mean size (in diameter) of $16.5 \mu \mathrm{m}$ for Gr. 1 and $26.5 \mu \mathrm{m}$ for Gr. 4 and typical rolling texture, in which the c-axis of hep unit cell is relatively inclined from normal direction (ND) of the plates to the TD. The texture distributions indicate that the basal pole in Gr. 4 is more inclined to the TD axis compared with Gr. 1.

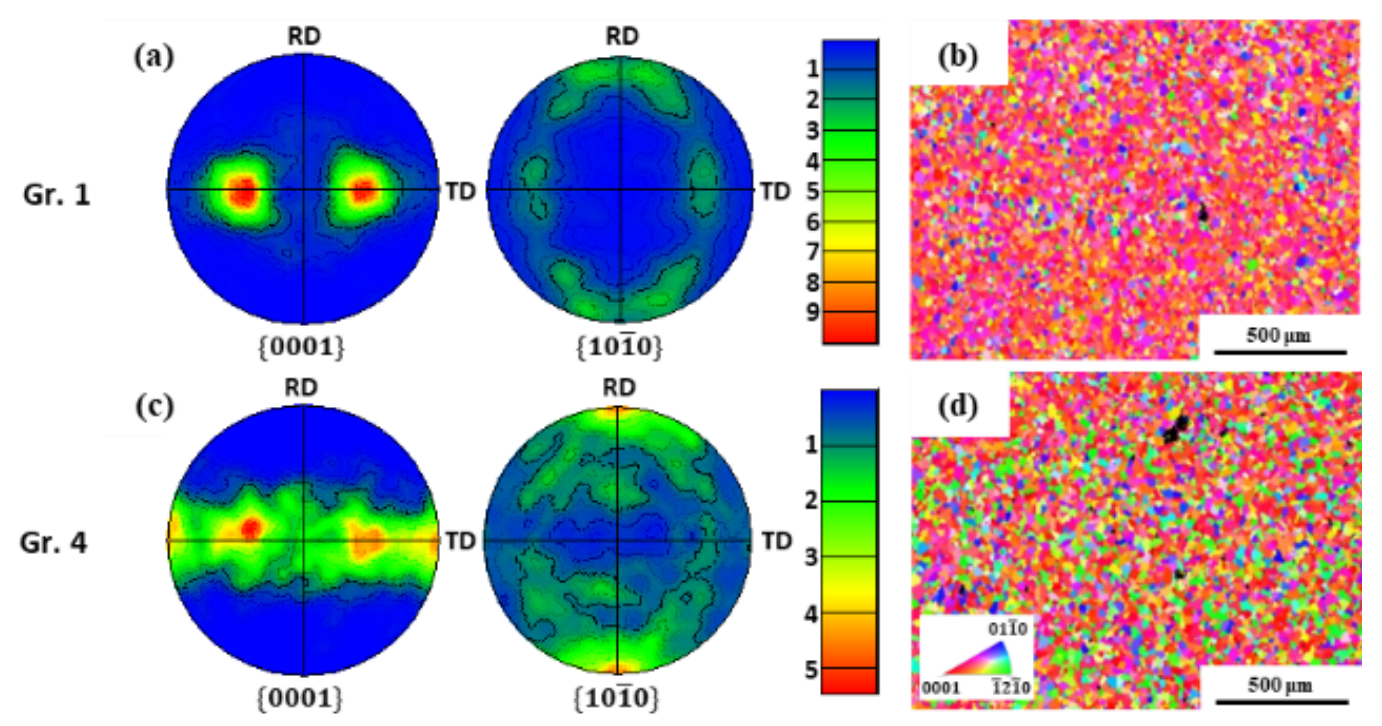

Figure 4. Pole figure (PF) showing the initial texture of (a) Gr. 1 and (c) Gr. 4 and inversed pole figure (IPF) maps of (b) Gr. 1 and (d) Gr. 4.

Figure 5 shows the different deformation behaviour with respect to the oxygen contents and the loading direction. The resultant tensile strength from both CSR method and SRJ method is higher in Gr. 4 than Gr. 1, and along the TD than RD. The higher strength in Gr. 4 could be explained by the solute strengthening effect by oxygen solute. The deformation behaviour of $\alpha$-Ti is typically anisotropic at grain scale, where the grain is softer in relation that loading direction is more parallel to the basal pole, whereas harder when the loading direction is more perpendicular. In this study, the tensile deformation along the TD is likely to more resemble with the latter relation inferred from the texture analysis. This explains the higher strength along the TD than RD.

(a)

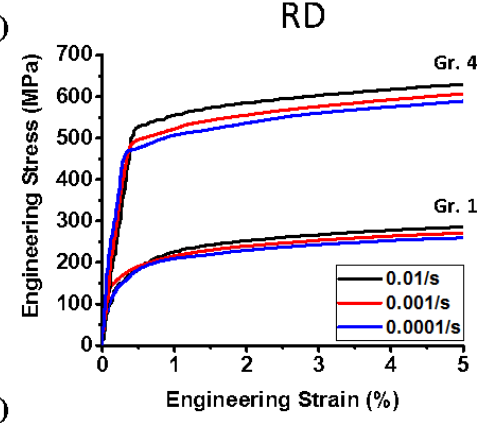

(c)

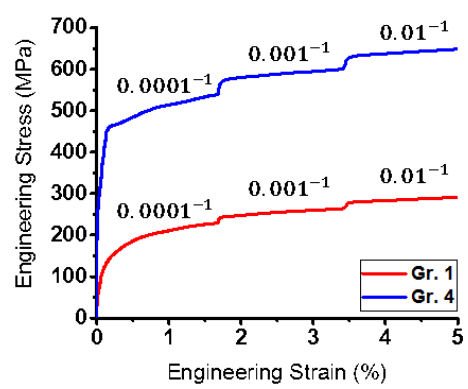

(b)
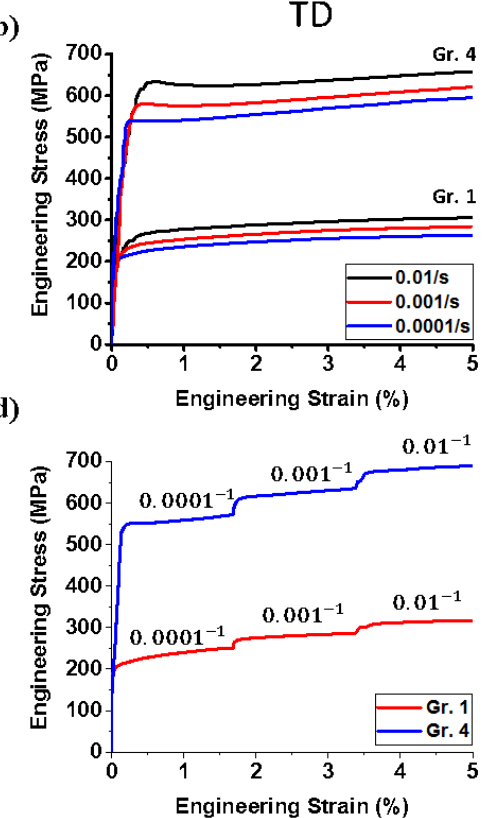

Figure 5. Engineering stress-strain curves resulting from the tensile test with CSR method along the (a) RD and (b) TD, and with SRJ method along the (c) RD and (d) TD.

The global SRS exponent, $m$ according to Eq. (1), was calculated with the flow stresses at 3\%, 4\% and 5\% in CSR method and 1.5\%,3\%, and 4.5\% in SRJ method. The $m$ values plotted in Figure 6 are tabled in Table 
1.

(a)

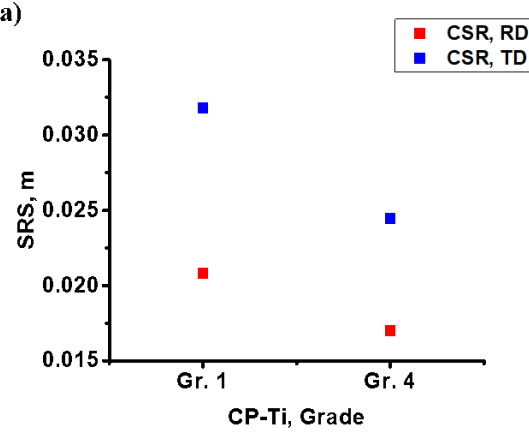

(b)

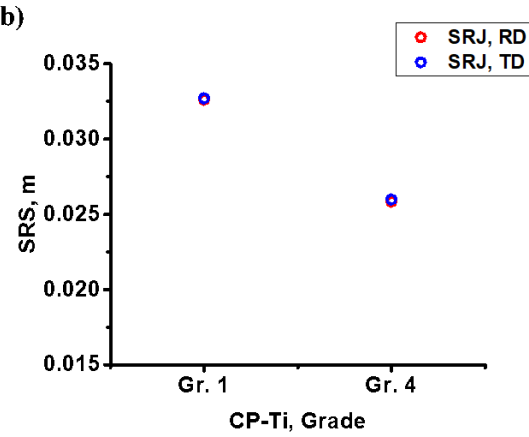

Figure 6. The global SRS exponent, m resulting from the macro-scopic tensile test with (a) CSR method and (b) SRJ method, respectively.

Table 1. The m value corresponding to the Figure 6.

\begin{tabular}{|c|c|c|c|c|}
\hline Grade & CSR-RD & CSR-TD & SRJ-RD & SRJ-TD \\
\hline Gr. 1 & 0.020 & 0.032 & 0.032 & 0.032 \\
\hline Gr. 4 & 0.017 & 0.024 & 0.025 & 0.026 \\
\hline
\end{tabular}

The global SRS is nearly independent on the loading direction in SRJ method, but not in CSR method, and there is an explicit dependence of SRS on the texture. Previous studies [4, 6] have provided an insight into the dependence of the SRS associated with the crystal orientation and corresponding slip activity in Ti. The active slip system of CP-Ti is thought to be different along the RD and TD loading direction due to the different texture distributions (see Figure 4). Schmid factor (SF) is thus analysed based on Figure 4(b) and (d) to anticipate the slip activity. The -prism and -basal slip system, most commonly activated in $\alpha$ phase Ti, are adopted for the SF analysis. Figure 7 shows that relatively higher SF for -prism and -basal slip system is found in the case of loading directions parallel to the RD and TD, respectively. Based on this SF analysis, the slip activity is likely to be different along the loading directions.

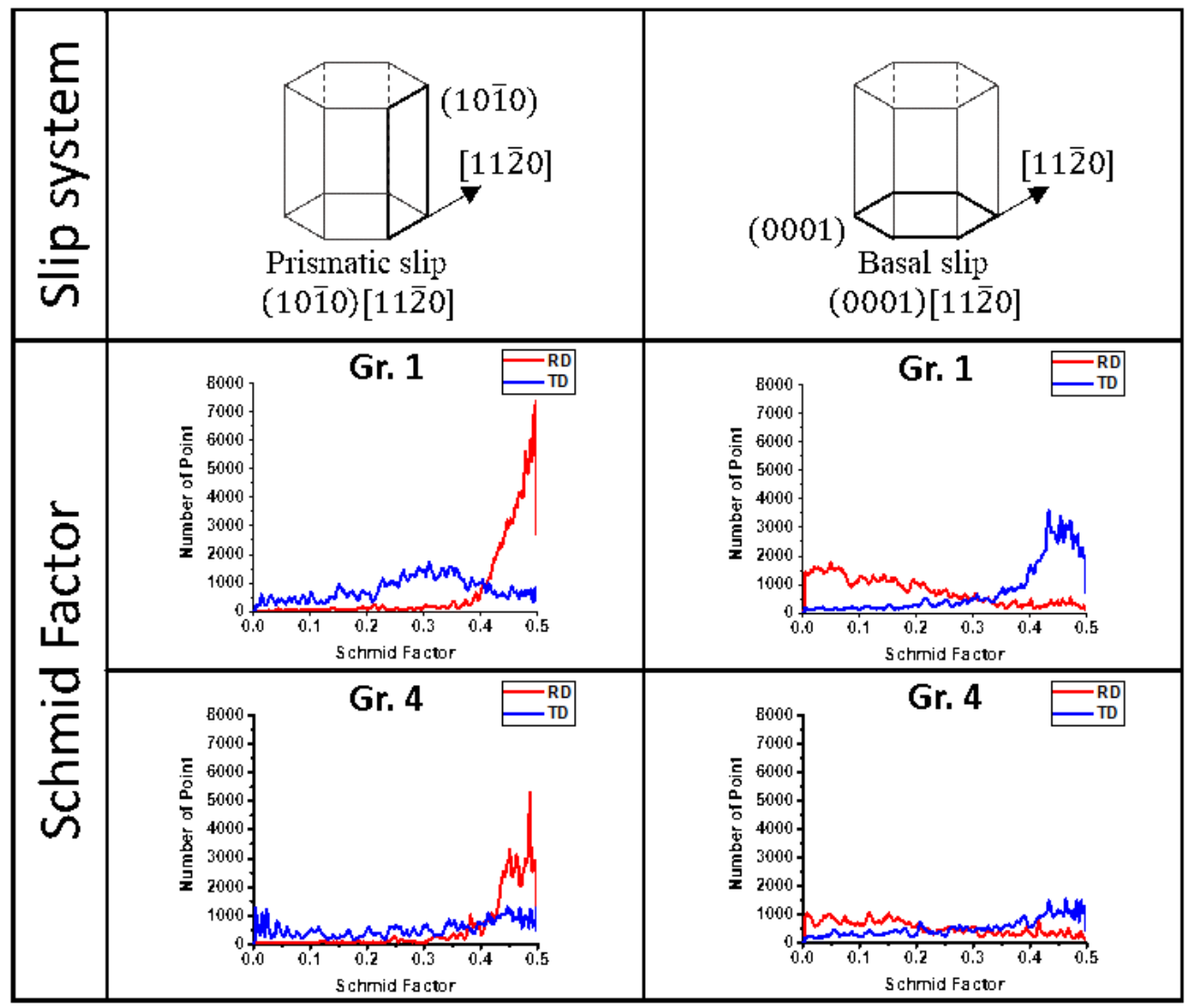


Figure 7. The Schmid factor analysis of CP-Ti along the RD and TD loading direction with respect to the -prism and -basal slip systems.

In addition to the deformation slip, twinning is an important deformation mode in Ti. The SRS is thought to be dependent on the twin activity in $\mathrm{Ti}$ [5]. For this reason, the twin activity of $\mathrm{CP}$-Ti is identified through the polarised light microscope. Figure 8 shows the representative images of specimens strained $\sim 5 \%$ with a strain rate of $10-2 \cdot \mathrm{s}-1$, and several twins are observed in Gr. 1, but not in Gr. 4 . The further specific analysis was done by EBSD measurement on the cross-section of Gr. 1 samples (see Figure 9), and the twin area fraction is compared along the loading directions (see Figure 10). This comparison shows the similar twin activity along the RD and TD. With the limited twin activity in Gr. 4, this result indicates that the influence of twinning on SRS is not significant in this work, and the texture dependent SRS agreed in CSR method is appropriate rather than SRJ method due to the significant difference of SF along the loading directions. A reason of texture independent SRS in SRJ method could be elucidtated by the significant hardening effect, especially significant at initial stage of deformation. Thus, further improved experimental design for the SRJ method is needed.

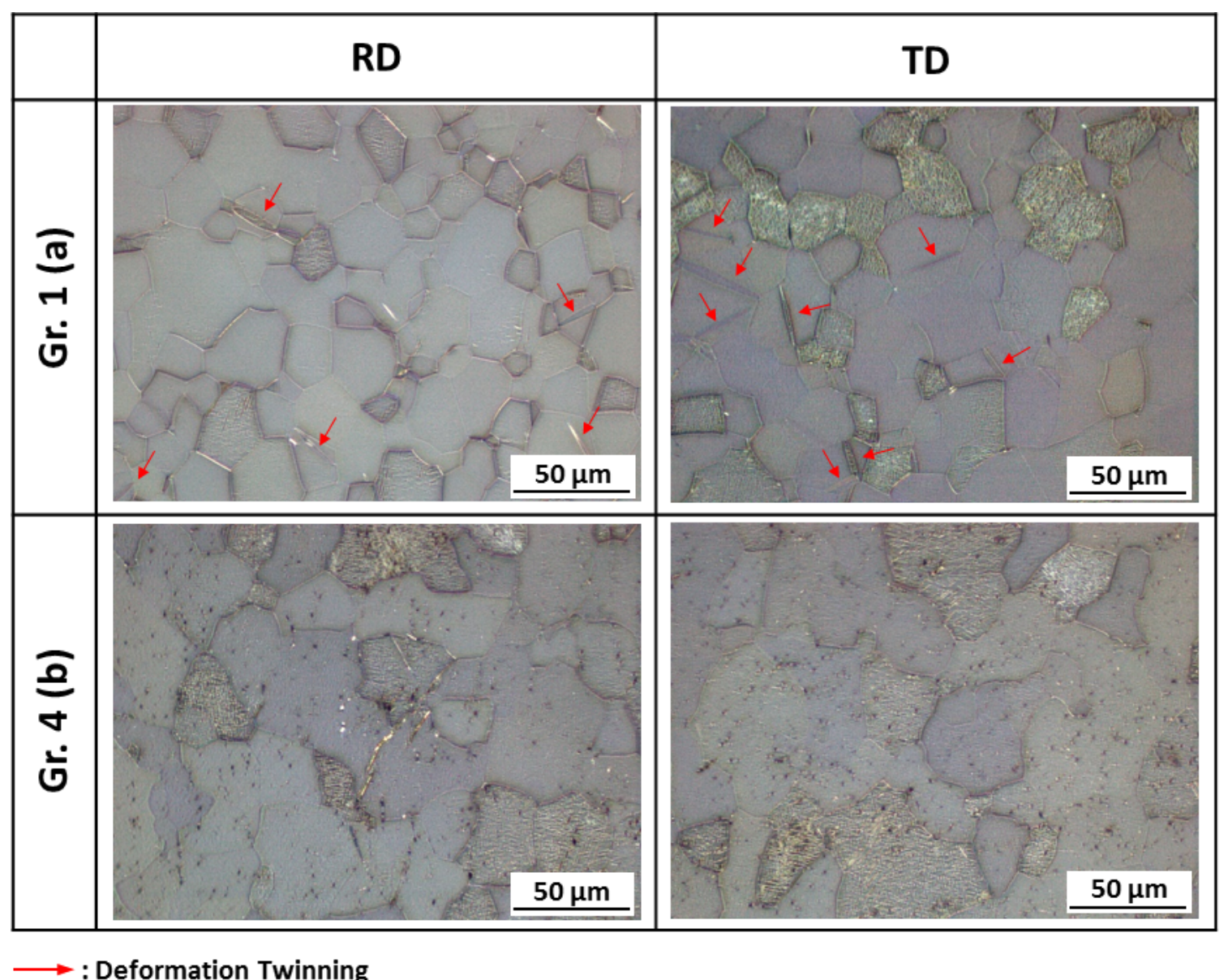

Figure 8. Polarised light microscope images of (a) Gr. 1 and (b) 4 tensile specimen interrupted at strain of $5 \%$ after loaded along the RD and TD with strain rate of 10-2·s-1. 

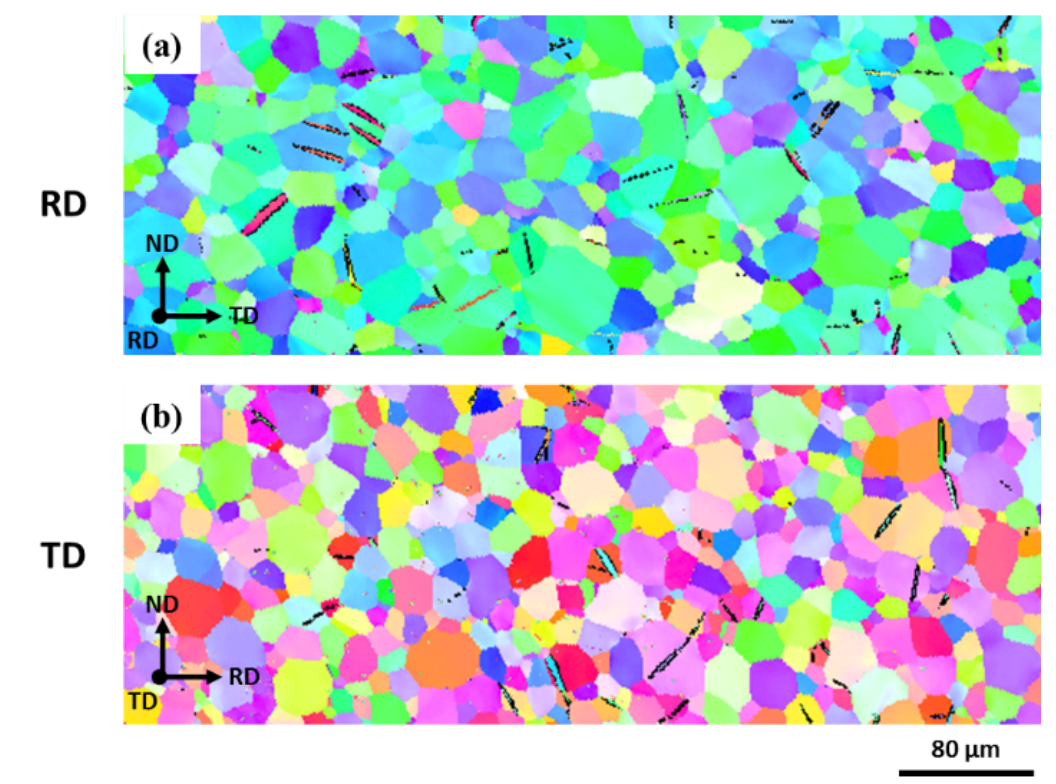

Figure 9. EBSD maps on the cross-section of Gr. 1 tensile specimens 5\%-strained with strain rate of 10-2-s-1 along the (a) RD and (b) TD. The twin boundaries are black-coloured.

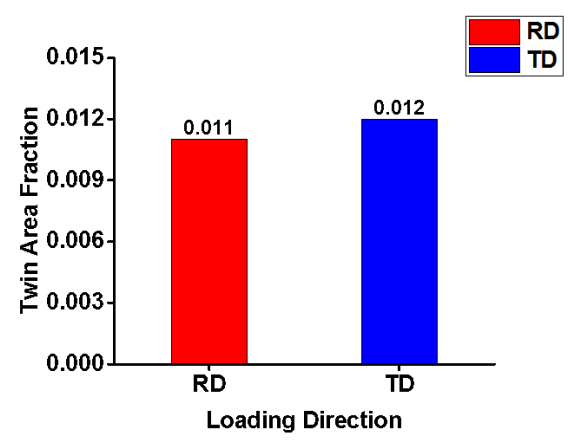

Figure 10. The twin area fraction analysed in the EBSD maps of Figure 9.

Although the design of experiment using SRJ method is somewhat inappropriate to evaluate the SRS dependent on the texture, the resultant SRS is likely to be a worthy finding in the influence of the oxygen contents with one of the CSR method. Our comparative investigation of SRS in Gr. 1 and 4 shows that the global SRS is higher in Gr. 1 than Gr. 4 (see Figure 6 and Table 1), and this effect of the oxygen contents is closely correlated with the findings in micro-scopic level.

The local SRS was evaluated with respect to a declination angle (i.e. angle between indent loading direction and c-axis of hcp unit cell) with three grain orientations (see Figure 11), which are relatively soft, intermediate and hard. The grain orientations of Gr. 1 are distributed on narrow range of the declination angle compared with Gr. 4, because the local SRS in Gr. 1 below the declination angle of $\sim 35^{\circ}$ was rather difficult to evaluate due to the frequent

displacement pop-in events by twin activity. Interestingly, the local SRS is dependent on the grain orientation regardless of the oxygen contents; the $m$ value is higher in softer grain.

The most striking finding in indentation test is that the SRS is lowered with decreasing the oxygen contents, and such influence of the oxygen contents is agreed with the result from the macro-scopic tests. This correlation in macro- and micro-scopic region is thought to complement the researches associated with rate sensitive deformation of $\mathrm{Ti}$ (e.g. dwell fatigue), and provide a clue to improve the performance of engineering components by appropriately controlling the oxygen solute and texture. 


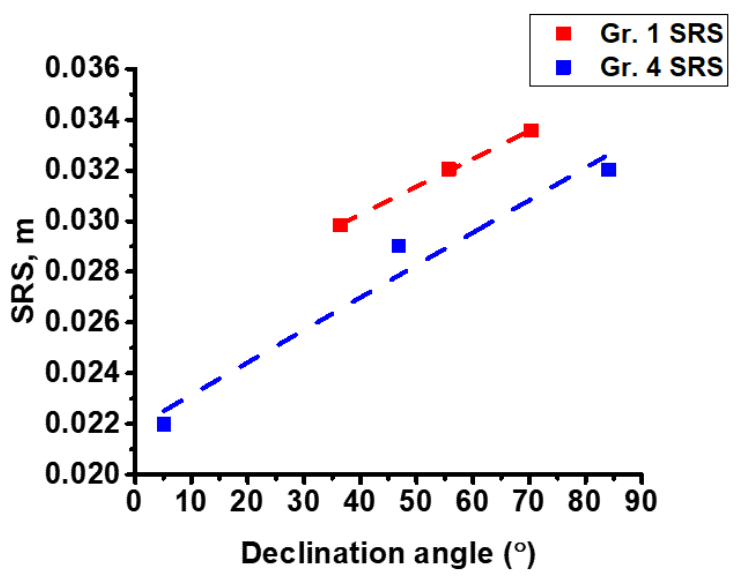

Figure 11. The local SRS exponent, m evaluated within the individual grain orientations of CP-Ti by nanoindentation test with SRJ method. The declination angle is the inclined extent of the c-axis of hep unit cell to the indent loading direction.

\section{Conclusion}

In this paper, we have examined the effect of oxygen contents on strain rate sensitivity (SRS) in comparative Gr. 1 and 4 CP-Ti, and in macro- and micro-scopic level by conducting the tensile test with CSM method and nanoindentation test with SRJ method. The slip activity anticipated by Schmid factor (SF) analysis is significantly different along the RD and TD loading directions, whereas the twin activity characterised by EBSD measurement is much similar. This suggest that the texture dependent global SRS (TD > RD) is closely related with the active slip systems. The anisotropic SRS is also found in grain scale as a function of declination angle, which is the inclined extent of c-axis in hcp structure to the indent loading direction; the local SRS is higher at relatively softer grain than harder grain. The most notable finding in present study is that the SRS is significantly dependent on their oxygen contents (Gr. $1>$ Gr. 4), and this dependence is clearly correlated in both macro- and micro-scopic level.

\section{Acknowledgements}

This research was supported by Basic Science Research Program through the National Research Foundation of Korea(NRF) funded by the Ministry of Education (No. 2017R1D1A1B03033424). We would like to thank Eun-Young Kim (KIMS) for assistance in accessing EBSD.

\section{Reference}

[1] M. Peters, J. Kumpfert, C. H. Ward and C. Leyens, Advanced Engineering Materials 5, (2003) 419-427.

[2] M. Bache, International journal of fatigue 25, (2003) 1079-1087.

[3] T.-S. Jun, D. E. Armstrong and T. B. Britton, Journal of Alloys and Compounds 672, (2016) 282-291.

[4] T.-S. Jun, Z. Zhang, G. Sernicola, F. P. Dunne and T. B. Britton, Acta Materialia 107, (2016) 298-309.

[5] Q. Luan, T. B. Britton and T.-S. Jun, Materials Science and Engineering: A 734, (2018) 385-397.

[6] Z. Zhang, T.-S. Jun, T. B. Britton and F. P. Dunne, Acta Materialia 118, (2016) 317-330.

[7] Z. Zhang and F. P. Dunne, Journal of the Mechanics and Physics of Solids 103, (2017) 199-220.

[8] V. Doquet and B. Barkia, Mechanics of Time-Dependent Materials 19, (2015) 153-166.

[9] G. Lütjering and J. C. Williams, Titanium, Springer Science \& Business Media (2007).

[10] W. L. Finlay and J. A. Snyder, JOM 2, (1950) 277-286.

[11] D.-s. Kang, K.-j. Lee, E.-p. Kwon, T. Tsuchiyama and S. Takaki, Materials Science and Engineering: A 632, (2015) 120-126.

[12] J.-M. Oh, B.-G. Lee, S.-W. Cho, S.-W. Lee, G.-S. Choi and J.-W. Lim, Metals and Materials International 17, (2011) 733-736.

[13] Z. Wyatt, W. Joost, D. Zhu and S. Ankem, International Journal of Plasticity 39, (2012) 119-131.

[14] Y. Wang and J. Huang, Materials Chemistry and Physics 81, (2003) 11-26.

[15] J. Gong and A. J. Wilkinson, Acta Materialia 57, (2009) 5693-5705.

[16] B. Barkia, V. Doquet, J.-P. Couzinié, I. Guillot and E. Héripré, Materials Science and Engineering: A 636, (2015) 91-102. 
[17] J. Williams, A. Sommer and P. Tung, Metallurgical Transactions 3, (1972) 2979-2984.

[18] T. S. Jun, Z. Zhang, F. P. Dunne and T. B. Britton, Proceedings of the 13th World Conference on Titanium, 498-498, Wiley Online Library,

[19] W. C. Oliver and G. M. Pharr, Journal of materials research 19, (2004) 3-20.

[20] B. Lucas and W. Oliver, Metallurgical and Materials Transactions A 30, (1999) 601-610.

[21] X. Li and B. Bhushan, Materials characterization 48, (2002) 11-36.

[22] V. Maier, K. Durst, J. Mueller, B. Backes, H. W. Höppel and M. Göken, Journal of Materials Research 26, (2011) 1421-1430.

[23] G. Welsch, R. Boyer and E. Collings, Materials properties handbook: titanium alloys, ASM international (1993).

[24] T. Britton, H. Liang, F. Dunne and A. Wilkinson, Proceedings 